\title{
Current advances on mechanisms and treatment of post-stroke seizures
}

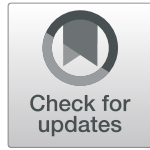

\author{
Yujiao Fu, Li Feng and Bo Xiao ${ }^{*}$ (D)
}

\begin{abstract}
Cerebrovascular diseases are among the most common causes of seizures in adults, especially in the elderly. With the increased incidence of stroke, the population with post-stroke seizures has grown, leading to the increased awareness of this disorder in the society. The most common seizure type after stroke is the focal seizure with or without evolution into bilateral convulsive seizures. Post-stroke seizures impair the quality of life, as well as the physical and mental health of those patients. Currently, the pathological and physical processes of post-stroke seizures are not quite clear yet. In this review, we summarize current advances in the pathogenesis, risk factors, and therapeutic targets of post-stroke seizures.
\end{abstract}

Keywords: Post-stroke seizures, Epilepsy, Pathogenesis, Treatment

\section{Background}

Post-stroke seizures are defined as seizures that occur after a stroke without a previous history of epilepsy. Epileptiform waves can be detected with electroencephalography (EEG) recording, and the location of epileptiform discharges is usually in accord with that of the stroke.

Based on the time of seizure onset, post-stroke seizures are referred to as early or late seizures. Early seizures occur $\leq 2$ weeks after the stroke, while late seizures occur $>2$ weeks after the insult. According to the definition by the International League Against Epilepsy, early seizures alone are not sufficient to make an epilepsy diagnosis as they are thought to be provoked, while a single late-onset seizure qualifies as symptomatic seizures due to the high risk of seizure recurrence within the next 10 years [1]. It has been reported that seizures occur in approximately $5 \%$ of subjects after ischemic stroke, of which one third occur within the first $24 \mathrm{~h}$ after stroke, and $50 \%$ within the first 30 days after stroke [2].

Recent prospective studies have demonstrated that $3.1-21.8 \%$ stroke patients develop post-stroke seizures

\footnotetext{
*Correspondence: xiaobo_xy@126.com

Department of Neurology, Xiangya Hospital, Central South University, Changsha 410000, China
}

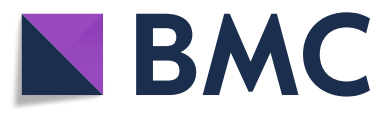

(- The Author(s). 2021 Open Access This article is licensed under a Creative Commons Attribution 4.0 International License, which permits use, sharing, adaptation, distribution and reproduction in any medium or format, as long as you give

appropriate credit to the original author(s) and the source, provide a link to the Creative Commons licence, and indicate if changes were made. The images or other third party material in this article are included in the article's Creative Commons licence, unless indicated otherwise in a credit line to the material. If material is not included in the article's Creative Commons licence and your intended use is not permitted by statutory regulation or exceeds the permitted use, you will need to obtain permission directly from the copyright holder. To view a copy of this licence, visit http://creativecommons.org/licenses/by/4.0/.
[3-5]. The wide range may be attributed to the sample size, the inclusion criteria, the definition of post-stroke seizures, and the duration of patient follow-up. Among the newly diagnosed epileptic patients over 60 years old, nearly $50 \%$ belong to the post-stroke epilepsy [3]. Meanwhile, the incidence of status epilepticus after stroke is less than $1 \%$. The incidence of post-stroke seizure using recently updated epilepsy definitions was proved to be similar to previous studies in a recent Swedish ischemic stroke cohort study [6]. Notably, Bentes et al. found that the post-stroke seizures are clinically underestimated since most seizures (92\%) were electroencephalographic without apparent clinical manifestations [4].

Seizures negatively affect stroke prognosis and the quality of life, in addition to the burdens of in-hospital costs. Thus, the diagnosis and treatment of this disorder are receiving much attention. Here, we will review the research development on post-stroke epilepsy for the purpose of advancing the understanding of both the prevention and treatment of this disorder.

\section{Pathogenesis of post-stroke seizures Pathogenesis of early seizures}

The early epileptic seizures have been demonstrated to be caused by local metabolic disorders and increased release of excitatory neurotransmitters, leading to the 
electrophysiological instability and neurotransmitter imbalance, which facilitate the onset of seizure attack.

\section{Electrophysiological instability}

The acute cerebral hemodynamic changes disrupt the local microenvironment, which reduces the neuronal membrane stability within the lesion, and causes excessive discharges. Ischemia and hypoxic insults are demonstrated to cause a sodium pump failure, which leads to increased intracellular $\mathrm{Na}^{+}$and cell depolarization. When the accumulation of $\mathrm{Na}^{+}$ reaches a certain extent inside the cell, calcium channels will be activated, leading to a sudden and quick influx of $\mathrm{Ca}^{2+}$ and thus a seizure attack [3]. In addition, neurons within the hippocampus, a region considered to play a crucial role in epileptogenesis, are also susceptible to abrupt ischemic insults and might become an epileptogenic focus [7]. Excessive neuronal excitation is also detected within the surrounding penumbra, which may be due to the disruped blood supply and hypoxia. Besides, reperfusion injury, hemorrhage and vasospasm are all reported to increase the susceptibility to the seizure attack [3].

\section{Neurotransmitter imbalance}

The imbalance between excitatory and inhibitory neurotransmitters is another contributor to the early seizures. In the central nervous system, the excitatory neurotransmitter glutamate and the inhibitory gamma aminobutyric acid (GABA) can be converted into each other by certain enzymes. Upon acute disruption of the hemodynamics, excitatory transmitters such as glutamate are increased, while GABA is denatured. As a result, an increased membrane excitability is induced and finally results in a seizure attack [8]. Besides, elevated adrenaline and decreased levels of dopamine are found to impact the calcium influx and decrease the seizure threshold [9].

It has been reported that early epileptic seizures are less likely to recur compared to the late-onset poststroke seizures, due to the fact that the epileptogenic factors including ischemia, hypoxia, and brain edema at the early stage after stroke are alleviated as disease progresses. Therefore, the early post-stroke seizures are considered to be self-limited.

\section{Pathogenesis of late seizures}

The late-onset post-stroke seizures result from a combination of complex factors including genetic factor, hemodynamic changes, inflammation, neural network reconstitution, glial proliferation, and metabolic disturbance.

\section{Genetic factor}

It has been established that about $30 \%$ of epilepsy syndromes are inherited, but only a few studies have assessed the genetic roles in epileptogenesis after stroke. Yang et al. have reported that the overexpression of aldehyde dehydrogenase 2 (ALDH2) partially blocked the increased level of malondialdehyde, which reduces the neuron apoptosis in the late stage and inhibits epileptic seizures [10]. Other factors such as CD40/-1C/T polymorphism, REST, HCN1, miRNAs, and transcription factors like $\mathrm{mSin} 3 \mathrm{~A}$ are also proven to play a role in regulating epileptogenesis in the late stage after stroke [3].

\section{Neurovascular unit imbalance}

In the late stage, the neurovascular integrity is disrupted due to the changes of regional cerebral blood flow, disruption of the blood brain barrier integrity, and inflammation. The neurovascular unit is composed of endothelial cells, neurons, and glial cells. Disruptions of the structural and functional integrity of neurovascular unit result in imbalanced micro-environment around neurons, subsequently causing abnormal discharges and the occurrence of late post-stroke seizures [11].

\section{Discruption of the neuronal network}

The epileptic seizures have been defined as a neurologic disorder with neuronal networks involved, rather than a single pathological process. This definition also applies to the post-stroke seizures. The neural network consists of neural circuits, nerve fibers and synapses. The neural repair process occurs in the late stage after stroke, including neurogenesis, integration of new neuronal loops, and synaptic connections. However, maladaptive neurogenesis and synaptic connection may overactivate the neural circuits, thereby increasing the seizure susceptibility [12].

\section{Glial activation}

The post-stroke glial proliferation and activation also play a role in epileptogenesis. In the late stage, the proliferation and activation of astrocytes in the cortex may lead to dysfunctions of ion channels, leading to cell depolarization, increased glutamate, and decreased GABA, all of which contribute to the late post-stroke seizure attack [13]. Particularly, the rise in glutamate levels in the setting of acute stroke was suggested as a potential clinically relevant biomarker for the development of post-stroke epilepsy [14]. In addition to astrocytes, oligodendrocytes and microglia may also proliferate after stroke, though their relations with epileptogenesis remain to be explored. 


\section{Risk factors for post-stroke seizures}

The onset of post-stroke seizures is associated with a series of complex factors, including the type of stroke (ischemic or hemorrhagic), the location and size of the lesion, the severity of the disease, etc.

Observational studies have revealed distinct risk factors for early and late seizures. Intracerebral hemorrhage or cerebral infarction with hemorrhagic transformation, a higher stroke severity or high National institutes of health stroke scale (NIHSS) score, and alcoholism are associated with an increased risk of developing early seizures, while a significant cortical involvement and a higher stroke severity indicate a greater probability of late-onset seizures. A cohort study involving 469 stroke patients conducted from 1982 to 2003 with 20 years of follow up analyzed the incidence of seizures following stroke, and the results confirmed that moderate and severe disability was predictive of post-stroke seizure. No seizure occurred after, for example, lacunar stroke [2]. Besides, the valproic acid monotherapy and the lateonset of seizures have also been reported to be risk factors for post-stroke seizures. However, a meta-analysis which included studies published during 1990-2014, found no significant difference in the probability of late seizure episode between patients with cerebral hemorrhage and those with infarction; neither was gender proved to be the risk factor [15].

Recently, the therapeutic method for stroke has been found to be related with the incidence of post-stroke seizures. The development of reperfusion therapies, including intravenous tissue plasminogen activator (IV-tPA) and endovascular (intra-arterial therapy, IAT) thrombectomy, has caused a $68 \%$ increase in the number of ischemic stroke survivors [16]. However, since the endogenous tissue plasminogen activator (tPA) can sensitize the brain via up-regulation of the N-methyl-Daspartate receptors, which leads to a lower threshold for a hyperexcitable state, the application of r-tPA may also increase the risk of post-stroke seizures. A retrospective cohort study, which recruited 1357 in-hospital patients between 2008 and 2015, found that compared to the medication care patients, there was a 3-fold increase of post-stroke seizures in patients treated with IV-tPA only, and a 5-fold increase in patients treated with IAT only [16]. Another study, however, reported that the incidence of post-stroke seizures was similar between the recombinant-tPA group and the naive group [17]. The discrepancy between the two studies might be explained by whether the analysis was adjusted for prognostic factors for seizure development.

Notably, Galovic et al. have developed a novel prognostic model for predicting late seizures after ischemic stroke, based on five independent risk factors. During the development and validation of a multivariable prediction model in the study, 1200 participants who had ischemic stroke in Switzerland were recruited. Finally, five variables were selected to generate the model, named as SeLECT (corresponding to Severity of stroke, Large-artery atherosclerosis, Early seizures onset, Cortical involvement, and Territory of middle cerebral artery involvement) [18]. For the severity of stroke, 0 for those with NIHSS scores $\leq 3,1$ for those with NIHSS scores between 4 and 10, 2 for those with NIHSS scores $\geq 11$; for the large-artery atherosclerosis, 0 for no, and 1 for yes; for the early seizure ( $\leq 7$ days), 0 for no, and 3 for yes; for the cortical involvement, 0 for no, and 2 for yes; for the territory of middle cerebral artery (MCA), 0 for no, and 1 for yes. Based on this model, a score of 0 was associated with a $0.7 \%$ risk of late seizures, while the highest score 9 predicted a $63 \%$ risk of late seizures within 1 year. The SeLECT score was validated in 1169 subjects from three independent international cohorts in Germany, Austria, and Italy, and showed good performance for prediction of late seizures after ischemic stroke $[18,19]$. However, more studies are needed to validate the reliability of the SeLECT model.

Apart from the risk factors for post-stroke seizures, the risk factors for seizure recurrence are also under investigations. One report showed that young age and male gender are highly suggestive of seizure recurrence after the first onset of post-stroke seizures [20]. Another study reported that a relatively large volume of hematoma is associated with seizure recurrence [21]. A recent hospital-based retrospective study involving 153 patients has identified that the presence of status epilepticus is a predictor for seizure recurrence after early seizures, the young age is indicative of seizure recurrence after late seizures, and the late onset of seizures is an independent predictor for seizure occurrence since earlyseizure patients are characterized by a lower likelihood of seizure recurrence and less frequent use of antiseizure medication (ASM) [22].

\section{Treatment of post-stroke seizures}

Upon the onset of post-stroke seizures, whether an ASM should be taken remains on debate. ASM are not recommended after stroke for the prevention of seizure attacks, since only one third of early seizure patients and half of late seizure patients would develop post-stroke seizures. A recent study in post-stroke epilepsy patients showed that $27.1 \%$ of patients who received ASM treatment after the first epileptic attack developed seizures 1 year later, while $53.8 \%$ of patients without ASM treatment did at the same time. Therefore, the authors concluded that ASM treatment should be recommended for post-stroke epilepsy patients [23]. However, when the ASM treatment should be given remains to be discussed. It is generally accepted that the ASM be taken after the 
second early seizure attack and the first late seizure attack to prevent the recurrence [16]. However, data from a clinical research demonstrated that early seizures often suggest poor clinical outcomes and increased mortality during hospitalization [3], suggesting that ASM treatment should be given after the first early seizure attack. In an intracerebral hemorrhage model, ASM treatment was found to be poorly associated with the prevention of epileptic attack [21].

Both classic (carbamazepine, valproate, phenytoin, and phenobarbital), and new ASM (oxarbazepine, lamotrigine, topiramate, levetiracetam, and etc.) can be used for patients with post-stroke epilepsy. The ASM exert antiepileptic effects via their exact mechanisms including sodium and calcium channel retardation, and regulation of the neurotransmitter imbalance. However, as the mechanisms of early and late seizures are distinct and the pathophysiological change of early seizures is considered to be reversible, it is important to choose appropriate ASM for early and late epilepsies. Phenobarbital has been reported to reduce seizure attacks after stroke in mice [24]. Valproic acid has been shown to reduce poststroke seizures by inhibiting microglial activation and down-regulating inflammatory mediator release in rat models [25]. A nationwide multi-center questionnaire survey on the management and treatment of post-stroke epilepsy in Japan found that carbamazepine is the most commonly used ASM for post-stroke seizures, followed by valproate and levetiracetam [26]. Recently, compared with the traditional ASM, new ASM are more frequently used with less side effects and fewer drug interactions. A large nationwide study showed that for late-seizure patients, valproic, carbamazepine and new ASM (including oxcarbazepine, topiramate, levetiracetam, and pregabalin) performed better in controlling seizure attacks than phenytoin [27]. Topiramate was proved to be neuroprotective for post-stroke seizures due to its function to reduce the excitatory effects of glutamate and enhance GABA-mediated inhibition [28]. Levetiracetam has been suggested as an alternative for traditional ASM against post-stroke seizures, based on safety and efficacy profiles in clinical studies [29].

Some other drugs have also been reported to have antiepileptic effects. HMG-GoA reductase inhibitors (statins) were reported to reduce the expression of eNOS, increase the permeability of blood brain barrier, and decrease the expression of Bax, p53, Caspase-3, and inflammatory mediators, thus reducing seizures [30]. Study has shown that statins could reduce the incidence of post-stroke seizures, which might be attributed to the inhibitory effect on glial proliferation [31]. Statin was also proved to reduce the risk of post-stroke seizure, especially with intensive $[32,33]$. Mild hypothermia was also reported to reduce the risk of developing post- stroke seizures, together with down-regulations of Notch 3 and Notch 4 [34].

\section{Conclusion and future perspective}

The post-stroke seizures negatively affect the prognosis of stroke, including the length of stay, disability rate and mortality. It is generally accepted that early seizures have a greater impact on patients' prognosis than late seizures do. Various models of post-stroke seizures provide explanations for the pathogenesis and physiological process of epilepsy. However, it is more likely that a combination of factors contributes to the post-stroke seizure attacks. The mechanisms of post-stroke seizures remain to be clarified, and further preclinical and clinical research is needed to find out new targets for the treatment and prevention of post-stroke seizures.

\section{Abbreviations \\ @@@EEG: Electroencephalography; GABA: Gamma aminobutyric acid; tPA: Tissue plasminogen activator; NIHSS: National institutes of health stroke scale; MCA: Middle cerebral artery; ASM: Antiseizure medication ; IAT: intra- arterial therapy; IV-tPA: intravenous tissue plasminogen activator; ALDH2: aldehyde dehydrogenase 2}

Acknowledgements

Not applicable.

Authors' contributions

FY drafted the manuscript for intellectual content, $F L$ and $X B$ revised the manuscript, and approved the final manuscript.

\section{Funding}

This work was supported by the National Natural Science Foundation of China (81701182 and 81501025), and the Omics-based Precision Medicine of Epilepsy being entrusted by Key Research Project of the Ministry of Science and Technology of China (2016YFC0904400).

Availability of data and materials Not applicable.

\section{Declarations}

Ethics approval and consent to participate Not applicable.

Consent for publication

All authors gave consent to publication of this review.

\section{Competing interests}

Author Bo Xiao is a member of the Editorial Board for Acta Epileptologica. Author Bo Xiao was not involved in the journal's review of, or decisions related to this manuscript. None of the other authors have any conflicts of interests to disclose.

Received: 26 October 2020 Accepted: 31 May 2021

Published online: 05 July 2021

\section{References}

1. Beghi E, Carpio A, Forsgren L, Hedorffer D, Malmgren K, Sander J, et al. Recommendation for a definition of acute symptomatic seizure. Epilepsia. 2010;51(4):671-5. https://doi.org/10.1111/j.1528-1167.2009.02285.x.

2. Stefanidou M, Das Rohit R, Beiser A, Banu S, Kelly-Hayes M, Kase C, et al. Incidence of seizures following initial ischemic stroke in a community-based cohort: the framingham heart study. Seizure. 2017:47:105-10. 
3. Yang H, Rajah G, Guo A, Wang Y, Wang Q. Pathogenesis of epileptic seizures and epilepsy after stroke. Neurol Res. 2018:1-7. https://doi.org/10.1 080/01616412.2018.1460701.

4. Bentes C, Martins H, Peralta Ana R, Casimiro C, Morgado C, Franco A, et al. Post-stroke seizures are clinically underestimated. J Neurol. 2017;264(9): 1978-85. https://doi.org/10.1007/s00415-017-8586-9.

5. Castro Conde JR, Fuentes IQ, Campo CG, Sosa AJ, Millan BR, Exposito SH. EEG findings and outcomes of continuous video-EEG monitoring started prior to initiation of seizure treatment in the perinatal stroke. Early Hum Dev. 2018:120:1-9.

6. Hassani M, Cooray G, Sveinsson O, Cooray C. Post-stroke epilepsy in an ischemic stroke cohort-incidence and diagnosis. Acta Neurol Scand. 2020; 141(2):141-7. https://doi.org/10.1111/ane.13174.

7. Hwang JY, Aromolaran KA, Zukin RS. Epigenetic mechanisms in stroke and epilepsy. Neuropsychopharmacol. 2013;38(1):167-82. https://doi.org/10.103 8/npp.2012.134

8. Xie WJ, Dong M, Liu Q, Meng HM. Early predictors and prevention for poststroke epilepsy: changes in neurotransmitter levels. Transl Neurosci. 2016; 7(1):1-5. https://doi.org/10.1515/tnsci-2016-0001.

9. Bameri B, Shaki F, Ahangar N, Ataee R, Samadi M, Mohammadi H. Evidence for the involvement of the dopaminergic system in seizure and oxidative damage induced by tramadol. Int J Toxicol. 2018;37(2):164-70. https://doi. org/10.1177/1091581817753607.

10. Yang $H$, Song Z, Yang $G$, Zhang B, Chen M, Wu T, et al. The ALDH2 rs671 polymorphism affects post-stroke epilepsy susceptibility and plasma 4-HNE levels. PLoS One. 2014;9(10):e109634. https://doi.org/10.1371/journal.pone.01 09634.

11. Avellaneda-Gómez C, Rodríguez CA, Giralt SE, Gómez GA, Serra MM, Ceballos $P$, et al. Description of stroke mimics after complete neurovascular assessment. Neurologia. 2019;34(1):7-13. https://doi.org/10.1016/j.nrl.2016.10. 006.

12. Ansari AH, Cherian P, Caicedo A, Naulaers G, De VM, Van HS, et al. Neonatal Seizure Detection Using Deep Convolutional Neural Networks. Int J Neural Syst. 2018;29(4):1850011.

13. Boison D, Steinhäuser C. Epilepsy and astrocyte energy metabolism. Glia. 2018;66(6):1235-43. https://doi.org/10.1002/glia.23247.

14. Nicolo JP, O'Brien TJ, Kwan P. Role of cerebral glutamate in post-stroke epileptogenesis. Neurolmage Clin. 2019;24:102069. https://doi.org/10.1016/j. nicl.2019.102069.

15. Zhang C, Wang X, Wang Y, Zhang J, Hu W, Ge M, et al. Risk factors for poststroke seizures: a systematic review and meta-analysis. Epilepsy Res. 2014; 108(10):1806-16. https://doi.org/10.1016/j.eplepsyres.2014.09.030.

16. Naylor J, Thevathasan A, Churilov L, Guo R, Xiong Y, Koome M, et al. Association between different acute stroke therapies and development of post stroke seizures. BMC Neurol. 2018;18(1):61. https://doi.org/10.1186/s12 883-018-1064-x.

17. Lekoubou A, Awoumou JJ, Kengne AP. Incidence of seizure in stroke patients treated with recombinant tissue plasminogen activator: a systematic review and meta-analysis. Int J Stroke. 2017;12(9):923-31. https:// doi.org/10.1177/1747493017729239.

18. Galovic M, Döhler N, Erdélyi-Canavese B, Felbecker A, Siebel P, Conrad J, et al. Prediction of late seizures after ischaemic stroke with a novel prognostic model (the SeLECT score): a multivariable prediction model development and validation study. Lancet Neurol. 2018;17(2):143-52. https://doi.org/10.1016/S1474-4422(17)30404-0.

19. Galovic M, Döhler N, Erdélyi-Canavese B, Felbecker A, Siebel P, Conrad J, et al. The SeLECT score is useful to predict post-stroke epilepsy. Lancet Neurol. 2018;17(5):395-6. https://doi.org/10.1016/S1474-4422(18)3 0075-9.

20. Kim HJ, Park KD, Choi KG, Lee HW. Clinical predictors of seizure recurrence after the first post-ischemic stroke seizure. BMC Neurol. 2016;16(1):212. https://doi.org/10.1186/s12883-016-0729-6.

21. Neshige S, Kuriyama M, Yoshimoto T, Takeshima S, Himeno T, Takamatsu K, et al. Seizures after intracerebral hemorrhage; risk factor, recurrence, efficacy of antiepileptic drug. J Neurol Sci. 2015;359(1-2):318-22. https://doi.org/10.1 016/j.jns.2015.09.358

22. Tomari S, Tanaka T, Ihara M, Matsuki T, Fukuma K, Matsubara S, et al. Risk factors for post-stroke seizure recurrence after the first episode. Seizure. 2017:52:22-6. https://doi.org/10.1016/j.seizure.2017.09.007.
23. Oros MM, Smolanka V, Sofilkanich N, Borovik O, Luts V, Andrukh PG. Epilepsy after ishemic stroke: is it worth administering anticonvulsants after the first attack? Wiadomosci Lek. 2018;71(2):269-72.

24. Markowitz GJ, Kadam SD, Smith DR, Johnston MV, Comi AM. Different effects of high- and low-dose phenobarbital on post-stroke seizure suppression and recovery in immature CD1 mice. Epilepsy Res. 2011;94(3): 138-48. https://doi.org/10.1016/j.eplepsyres.2011.01.002.

25. Xuan A, Long D, Li J, Ji W, Hong L, Zhang M, et al. Neuroprotective effects of valproic acid following transient global ischemia in rats. Life Sci. 2012; 90(11-12):463-8. https://doi.org/10.1016/j.lfs.2012.01.001.

26. Gilad R. Management of seizures following a stroke: what are the options? Drugs Aging. 2012;29(7):533-8. https://doi.org/10.2165/11631540000000000-00000.

27. Huang YH, Chi N, Kuan Y, Chan L, Hu C, Chiou H, et al. Efficacy of phenytoin, valproic acid, carbamazepine and new antiepileptic drugs on control of late-onset post-stroke epilepsy in Taiwan. Eur J Neurol. 2015; 22(11):1459-68. https://doi.org/10.1111/ene.12766.

28. Doria JW, Forgacs PB. Incidence, implications and management of seizures following ischemic and hemorrhagic stroke. Curr Neurol Neurosci Rep. 2019. 19(7):37. https://doi.org/10.1007/s11910-019-0957-4.

29. Belcastro V, Costa C, Galletti F, Autuori A, Pierguidi L, Pisani F, et al. Levetiracetam in newly diagnosed late-onset post-stroke seizures: a prospective observational study. Epilepsy Res. 2008;82(2-3):223-610. https:// doi.org/10.1016/j.eplepsyres.2008.08.008.

30. Seker FB, Kilic U, Caglayan B, Ethemoglu M, Caglayan A, Ekimci N, et al. HMG-CoA reductase inhibitor rosuvastatin improves abnormal brain electrical activity via mechanisms involving eNOS. Neuroscience. 2015;284 349-59. https://doi.org/10.1016/j.neuroscience.2014.10.014.

31. Guo J, Guo J, Li J, Zhou M, Qin F, Zhang S, et al. Statin treatment reduces the risk of poststroke seizures. Neurology. 2015;85(8):701-7. https://doi.org/1 0.1212 WNL.0000000000001814.

32. Li Y, Zhang B, Zhang L, Xie D, Li Y. Efficacy of statin therapy in post-stroke seizure prophylaxis: clues from an observational study of routine secondary prevention treatment. Seizure. 2019;71:185-9. https://doi.org/10.1016/j. seizure.2019.07.010.

33. Vitturi BK, Gagliardi RJ. The influence of statins on the risk of post-stroke epilepsy. Neurol Sci. 2020;41(7):1851-7. https://doi.org/10.1007/s10072-02004298-5.

34. Yang GS, Zhou X, An X, Liu X, Zhang Y, Yu D, et al. Pathol Res Pract. 2018; 214(7):1008-16. https://doi.org/10.1016/j.prp.2018.05.003.
Ready to submit your research? Choose BMC and benefit from:

- fast, convenient online submission

- thorough peer review by experienced researchers in your field

- rapid publication on acceptance

- support for research data, including large and complex data types

- gold Open Access which fosters wider collaboration and increased citations

- maximum visibility for your research: over $100 \mathrm{M}$ website views per year

At $\mathrm{BMC}$, research is always in progress.

Learn more biomedcentral.com/submissions 